K. H. LEE, B. LEE, K. R. LEE, M. H. YI, N. H. HUR* (SOGANG UNiVERSity, SEOUL, KOREA) Dual Pd and $\mathrm{CuFe}_{2} \mathrm{O}_{4}$ Nanoparticles Encapsulated in a Core/Shell Silica Microsphere for Selective Hydrogenation of Arylacetylenes

Chem. Commun. 2012, 48, 4414-4416.

\title{
Dual $\mathrm{Pd}$ and $\mathrm{CuFe}_{2} \mathrm{O}_{4}$ Nanoparticles in a Core/Shell Silica Microsphere
}

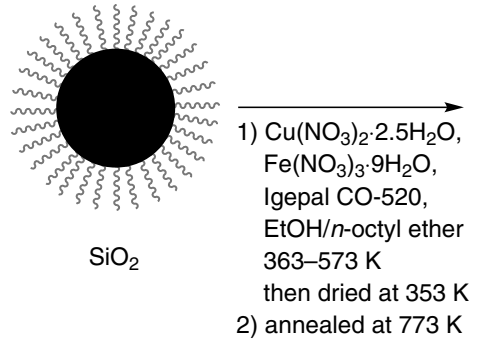

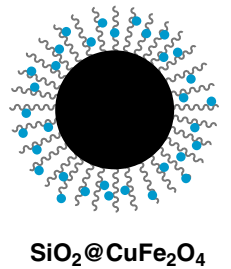

1) APTMS, EtOH then dried at $353 \mathrm{~K}$ 2) $\mathrm{K}_{2} \mathrm{PdCl}_{4}, \mathrm{H}_{2} \mathrm{O}$ then dried at $353 \mathrm{~K}$

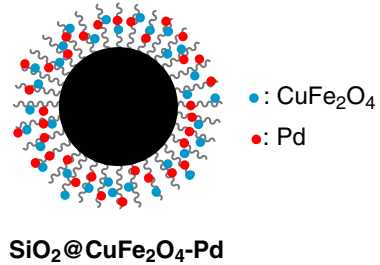

average diameter: $380 \mathrm{~nm}$

Igepal CO-520: 4- $\left(\mathrm{C}_{9} \mathrm{H}_{19}\right) \mathrm{C}_{6} \mathrm{H}_{4} \mathrm{O}\left(\mathrm{CH}_{2} \mathrm{CH}_{2} \mathrm{O}\right)_{4} \mathrm{CH}_{2} \mathrm{CH}_{2} \mathrm{OH}$

APTMS: 3-aminopropyltrimethoxysilane<smiles>C#Cc1ccccc1</smiles>

\section{$\mathrm{SiO}_{2} @ \mathrm{CuFe}_{2} \mathrm{O}_{4}-\mathrm{Pd}(10 \mathrm{mg})$}

hexane $(2 \mathrm{~mL}), \mathrm{H}_{2}$ balloon

(0.91 mmol)<smiles>C=Cc1ccccc1</smiles><smiles>C=Cc1ccccc1C(F)(F)F</smiles><smiles>C=Cc1ccc(F)cc1</smiles><smiles>C=Cc1ccc(C)cc1</smiles>

$98 \%$ conversion $98 \%$ selectivity TOF $90 \mathrm{~h}^{-1}$

$99 \%$ conversion $88 \%$ selectivity TOF $203 \mathrm{~h}^{-1}$

$99 \%$ conversion $98 \%$ selectivity TOF $226 \mathrm{~h}^{-1}$

$99 \%$ conversion $73 \%$ selectivity TOF $84 \mathrm{~h}^{-1}$
Significance: A dual catalyst containing $\mathrm{Pd}$ and $\mathrm{CuFe}_{2} \mathrm{O}_{4}$ nanoparticles in a core/shell silica microsphere $\left(\mathrm{SiO}_{2} @ \mathrm{CuFe}_{2} \mathrm{O}_{4}-\mathrm{Pd}\right)$ for selective hydrogenation of arylacetylenes to styrenes was described. A sequential modification of $\mathrm{SiO}_{2}$ with $\mathrm{CuFe}_{2} \mathrm{O}_{4}$ and $\mathrm{Pd}$ nanoparticles led to the formation of the dual catalyst $\mathrm{SiO}_{2} @ \mathrm{CuFe}_{2} \mathrm{O}_{4}-\mathrm{Pd}$. The hydrogenation of arylacetylenes was performed in hexane under $\mathrm{H}_{2}$ (1 atm) using $\mathrm{SiO}_{2} @ \mathrm{CuFe}_{2} \mathrm{O}_{4}-\mathrm{Pd}$ to give the corresponding styrenes in 98-99\% conversion with 73-98\% selectivity.

\section{Gategory}

Polymer-Supported Synthesis

Key words

dual catalysts

nanoparticles

selective

hydrogenation

heterogeneous catalysis
Comment: $\mathrm{SiO}_{2} @ \mathrm{CuFe}_{2} \mathrm{O}_{4}-\mathrm{Pd}$ was prepared on the basis of the authors' previous work (Chem. Mater. 2008, 20, 6738). The $\mathrm{SiO}_{2} @ \mathrm{CuFe}_{2} \mathrm{O}_{4}-\mathrm{Pd}$ catalyst was recovered by using an external magnet and reused in the hydrogenation of phenylacetylene ( $1^{\text {st }}$ use: $98 \%$ conversion, $98 \%$ selectivity; $2^{\text {nd }}$ use: $99 \%$ conversion, $98 \%$ selectivity; $3^{\text {rd }}$ use: $98 \%$ conversion, $97 \%$ selectivity). $\mathrm{SiO}_{2} @ \mathrm{CuFe}_{2} \mathrm{O}_{4}-\mathrm{Pd}$ was characterized by powder $X$-ray diffraction, TEM, EDX, HR-SEM, ICP, fielddependent magnetization, etc. 